\title{
Simultaneous Optimization of Tuning PID Cascade Control System Using Duelist Algorithms
}

\author{
Totok Ruki Biyanto* \\ Engineering Physics, Institut Teknologi Sepuluh Nopember \\ Process Design, Control and Optimization Laboratory \\ Surabaya, Indonesia \\ trb@ep.its.ac.id
}

Abu Hasan

General Manajer

PT. PJB UP Paiton

Paiton, Probolinggo, Indonesia

Abu.hasan@ptpjb.com

\author{
Muhammad Salman Alfarisi \\ Engineering Physics, Institut Teknologi Sepuluh Nopember \\ Process Design, Control and Optimization Laboratory \\ Surabaya, Indonesia \\ muhammad.salman12@mhs.ep.its.ac.id
}

\author{
Hendrik Setiawan \\ Instrumentation and Control Department \\ PT. PJB UP Paiton \\ Paiton, Probolinggo, Indonesia \\ hendrik.s@ptpjb.com
}

\author{
Naindar Afdanny \\ Engineering Physics, Institut Teknologi Sepuluh Nopember \\ Process Design, Control and Optimization Laboratory \\ Surabaya, Indonesia \\ afdanny14@mhs.ep.its.ac.id
}

\begin{abstract}
In this research, Proportional-Integral-Derivative (PID) controller was integrated into the advanced control system to achieve an optimal performance. One type of advanced control system is cascade control. In conventional cascade control, controller parameters in primary and secondary loops are tuned sequentially. First, the parameters in secondary controller are tuned using certain tuning rule, meanwhile the primary loop in the open loop mode. Furthermore, the controller parameters in primary loop are tuned by considering the influence of the controlled secondary loop. This makes the tuning of PID cascade control more difficult and takes more time consuming. This paper describes a method of simultaneous tuning cascade control system using Duelist Algorithm (DA) to estimate the proper control parameters of the primary and the secondary loops in the plants. The analysis was conducted based on the evaluation of the closedloop response to a step set point changes. The results show that the proposed method has better control performance than the previous method. PID auto tuning using DA provide more robust performance of the control system.
\end{abstract}

Keywords—cascade control; auto tuning; PID; duelist algorithm

\section{INTRODUCTION}

A requirement of the control system in the industry is the achievement of satisfactory performance and ease of setup. PID controller is commonly used in an industrial process control to obtains good performance with a simple algorithm. It is easy to be understood by operator and easy to applied in all industrial application [1]. Commonly, PID controller is integrated into an advanced control system to achieve better performance.
One type of advanced control systems is cascade control. The purpose of cascade control is to accelerate system response $[2,3]$. Cascade control is widely used in industrial processes. It caused by effectiveness, robustness, and simple design to apply [2]. Cascade control system consists of the primary loop (outer) and the secondary loop (inner). It has two feedback loops, which the output of the primary loop controller will represent as the set point in the secondary loop. The signal output from the secondary loop controller is directly to the actuator such as control valve or pump [3].

Although the scheme has been developed more sophisticated [4-6], the standard scheme of control cascade consists of two loops with two PID controllers. In conventional cascade control, controller parameters in primary and secondary loops are tuned sequentially. First, the parameters in secondary controller are tuned using certain tuning rule, meanwhile the primary loop in open loop state. Furthermore, the controller parameter in primary loop are tuned by considering the influence of the controlled secondary loop. If the results obtained are not satisfactory, there will be a repetition [7]. This makes the tuning of PID cascade control more difficult and takes more time consuming. Some researches on the simultaneous cascade control system have been carried out [8-11]. In PID cascade control, there are 6 parameters to be determined. Thus, it needs an optimization method to estimate the proper control parameters of the primary and secondary loops.

The application of stochastic optimization algorithm in control system have been used previously. Recently, Genetic Algorithm (GA) has been utilized for tuning PID cascade control system [12]. In this research, the new stochastic 
optimization algorithm is used to improve tuning in both PID controller parameters on the cascade control system, simultaneously. The new stochastic algorithm is Duelist Algorithm (DA). DA is algorithm that mimic a fight. In DA, the different treatment has been given to a fighter based on the results of the fight, win or lose. The fighter who lost could learn from the winners, meanwhile the winners attempt some new capabilities to improve his fighting abilities [13]. It is known that the DA showed some better results compared to other methods: GA, Particle Swarm Optimization Algorithm (PSO), and Imperialist Competitive Algorithm. DA obtains the achievement of global optimization with the better and faster iterations.

\section{CAScAde Control System}

In this research, PID auto tuning using DA on cascade control system is designed and described in the following sub section.

\section{A. Riview Cascade Control}

Cascade control system is often applied in the industry. The purpose of cascade control system is to accelerate the response and to improve the dynamic performance of the system. Cascade control is widely used in industrial processes such as reactors, distillation columns, heat exchangers, fire heaters, etc. It is due to the effectiveness, robustness, and simple design to apply. Cascade control loop structure consists of the primary loop (outer) and the secondary loop (inner). It has two feedback loops. The output of the primary loop changes the set point in the secondary loop. The secondary loop located inside the primary control loop. The signal output from the secondary loop is directly to the actuator such as control valve or pump.

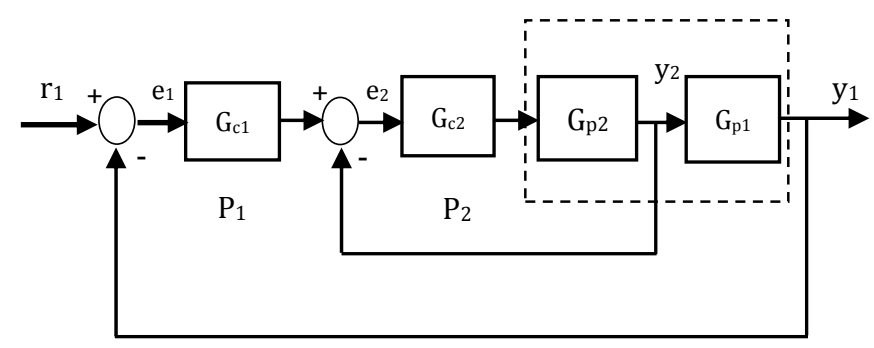

Fig. 1. Configuration of cascade control

The structure of cascade controller is shown in Fig. 1. The process consists of two components $\mathrm{G}_{\mathrm{p} 1}$ and $\mathrm{G}_{\mathrm{p} 2}$, represented by the dashed box. $G_{c 2}$ is the secondary controller and $G_{c 1}$ is the primary controller. In conventional cascade control, controller parameters in primary and secondary loops are tuned sequentially. First, the parameters in secondary controller are tuned using certain tuning rule, meanwhile the primary loop in the open loop mode. Furthermore, the controller parameters in primary loop are tuned by considering the influence of the controlled secondary loop. If the results obtained are not satisfactory, there will be a repetition. This makes the tuning of PID cascade control more difficult and takes more time consuming. The transfer functions of cascade control can be written as follows:

$$
\begin{aligned}
G_{y_{2} e_{2}} & =\frac{G_{p 2}(s) G_{c 2}(s)}{1+G_{p 2}(s) G_{c 2}(s)} \\
G_{y_{1} e_{1}} & =\frac{G_{p 1}(s) G_{c 1}(s) G_{y_{2} e_{2}}(s)}{1+G_{p 1}(s) G_{c 1}(s) G_{y_{2} e_{2}}}
\end{aligned}
$$

To complete the design of cascade control system, the parameters of two controllers have to be determined. In this research, the system used DA to find the parameters of two controllers that represented by $\mathrm{Kp}, \mathrm{Ki}, \mathrm{Kd}$ on primary controller and Kps, Kds, Kis on secondary controller.

\section{B. Design Plant and Controller}

The secondary and primary controllers are used PID controller. This model is simulated on Matlab. The principle of this proposed method tuning PID is simultaneously, where parameters of both PID controllers are tuned at the same time. The plant transfer function is assumed to be some order and tabulated on Table 1.

TABLE I. TRANSFER FUNCTION PLANT

\begin{tabular}{|c|l|}
\hline Zero Order Response System & $\frac{C(s)}{R(s)}=\frac{K p}{s}$ \\
\hline Zero Order with Time Delay & $\frac{C(s)}{R(s)}=\frac{K p e^{-\theta s}}{s}$ \\
\hline First Order Response System & $\frac{C(s)}{R(s)}=\frac{K p}{\tau s+1}$ \\
\hline First Order with Time Delay & $\frac{C(s)}{R(s)}=\frac{K p}{\tau s+1} e^{-\theta s}$ \\
\hline Second Order Response System & $\frac{C(s)}{R(s)}=\frac{K p}{\tau^{2} s^{2}+2 \zeta \tau s+1}$ \\
\hline Second Order with Time Delay & $\frac{C(s)}{R(s)}=\frac{K p e^{-\tau s}}{\tau^{2} s^{2}+2 \xi \tau s+1}$ \\
\hline
\end{tabular}

The primary and secondary controllers are assumed to be parallel PID controllers, respectively, which have the following forms.

$$
\begin{aligned}
& p(t)=K_{c}\left[\left[e(t)+\frac{1}{T_{i}} \int_{0}^{t} e(t) d t+T_{D} \frac{d e(t)}{d t}\right]\right. \\
& G_{c}(s)=K_{c}\left\lfloor 1+\frac{1}{T_{i} s}+T_{D} s\right\rfloor
\end{aligned}
$$

The development of the three types of control parameters achieved three gain, $K_{p}, K_{i}, K_{D}$, more than the standard parameters $K_{C}, T_{i}, T_{D}$. Development of this parameter is also used in Matlab. The equation is modeled in the following equation.

$$
p(t)=\bar{p}+K_{p} e(t)+K_{i} \int_{0}^{t} e(t) d t+K_{D} \frac{d e(t)}{d t}
$$




\section{OPTIMIZATION PARAMETERS}

The cascade control system is designed by some steps i.e. modelling of primary and secondary plants and build an algorithm to optimize controller parameters based on DA. By incorporating both model at optimizer, the optimization of six tuning parameters ( $\mathrm{Kp}, \mathrm{Ki}, \mathrm{Kd}, \mathrm{Kps}$, Kis, and $\mathrm{Kds}$ ) for each controller was performed. The optimization of six parameters are obtained by simulation in Matlab, in order to obtain the optimal objective function.

\section{A. Objective Function Evaluation}

Optimal tuning parameters is achieved, if the accumulated error or internal error criteria is minimum. One of the internal error is Integral Absolute Error (IAE) as shown in equation 7.

$$
I A E=\int_{0}^{\infty}|e(t)| d t=\int_{0}^{\infty}|r(t)-y(t)| d t
$$

In this research, IAE is obtained by changing the set point value using unit step and calculated the accumulated error up to settling time. IAE of primary loop controller is used as an objective function and to be minimized by DA. On the other hand, the performance analyses are evaluated based on error steady state, settling time, and maximum overshoot.

\section{B. Optimization Using Duelist Algorithm}

A new algorithm which is inspired by human fighting and learning capabilities called Duelist Algorithm (DA). The individual in population are called duelist. All those duelists would fight one by one to determine the champions, winners and losers. In DA, different treatment given to a duelist based on the results of the fight, win or lose. Duelist who lost will learn from the winners with copied binary array from the winners. The winners try new capabilities to improve his fighting abilities with change binary array on its body.

DA on m-files connected with Simulink to call modeling of the plant. The initial step in this tuning method is to determine the value of the initial program. That amount includes duelist population, the number of generations duelist, dimensions that will be optimized, as well as other value in the search for points of convergence.

The next step, DA will search optimal controller parameters which provide minimum IAE. More detail about the optimization of tuning parameters in the cascade control will be described as follows:

Step 1: Determine the objective function.

Step 2: Determine the DA optimization parameters is shown as follows.

Population $=20$

MaxGeneration $=50$

FightCapabilities $=20$

Champion $=0.1$

ProbLearning $=0.8$

ProbInnovate $=0.1$

Luckcoeff $=0.01$

Dimension $=6$
Step 3: Simulate the cascade control tuning parameter optimization to obtain minimal IAE.

Step 4: Analyze the control performances based on qualitative and quantitative method.

\section{RESULT AND DISCUSSION}

The plant models based on Sadisavarao's paper were utilized as in this research, and it is shown in the following equation [12].

$$
\begin{aligned}
& G p 1=\frac{e^{-10 s}}{100 s+1} \\
& G p 2=\frac{2 e^{-2 s}}{20 s+1}
\end{aligned}
$$

The simulation was performed over the time from initial steady state to next steady state in minutes. The performance of the proposed method was compared to the other previous cascade tuning method i.e. Zieger Nichols method, the method proposed by Lee et al., the method proposed by Luus \& Jaloka, and GA.

In the first scenario, the proposed and previous methods, the PID control mode is tuned in PI at primary loop and P only in secondary loop. The result comparisons of proposed and previous methods are tabulated in the Table 2.

TABLE II. PARAMETER PID USING PI-P

\begin{tabular}{|c|c|c|c|c|c|c|}
\hline Methods & Kp & Ki & Kd & Kps & Kis & Kds \\
\hline Lee et al. & 5.8300 & 0.0555 & - & 3.4440 & - & - \\
\hline Luus \& Jaloka & 4.6386 & 0.0464 & - & 2.8511 & - & - \\
\hline Zieger Nichols & 5.5700 & 0.1379 & - & 3.3500 & - & - \\
\hline GA & 6.7552 & 0.0672 & - & 5.8500 & - & - \\
\hline DA & 4.961 & 0.044 & - & 4.787 & - & - \\
\hline
\end{tabular}

By utilizing the tuning parameters in Table 2, the simulation result of cascade control in secondary and primary control can be shown in Fig. 2 and Fig. 3, respectively.

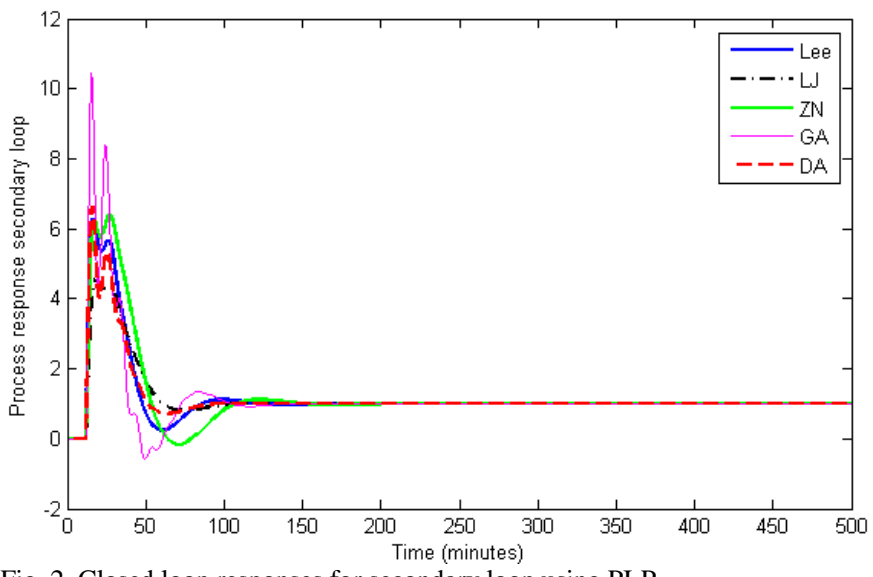

Fig. 2. Closed loop responses for secondary loop using PI-P 


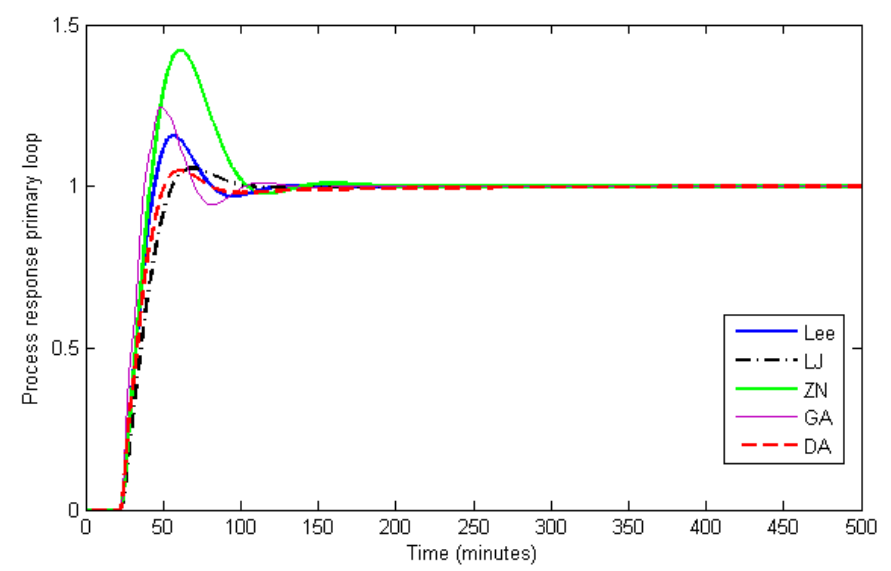

Fig. 3. Closed loop responses for primary loop using PI-P

From the Fig. 3, the quantitative performance (IAE) and qualitative performance of the system is tabulated on the Table 3. The result shown, tuning PID-DA surpass all previous method and it is shown in the smallest IAE, fastest settling time, and smallest overshoot.

TABLE III. PERFORMANCE COMPARISONS USING PI-P

\begin{tabular}{|l|c|c|c|c|}
\hline \multicolumn{1}{|c|}{ Metode } & IAE & $\begin{array}{c}\text { Error } \\
\text { steady state }\end{array}$ & $\begin{array}{c}\text { Settling } \\
\text { Time } \\
\text { (minutes) }\end{array}$ & $\begin{array}{c}\text { Overshoot } \\
(\%)\end{array}$ \\
\hline Lee et al. & 74.83 & $0.0 \%$ & 106.5 & $16.3 \%$ \\
\hline Luus \& Jaloka & 77.24 & $0.0 \%$ & 89.5 & $5.9 \%$ \\
\hline GA & 72.39 & $0.0 \%$ & 124 & $29.9 \%$ \\
\hline Zieger Nichols & 95.72 & $0.0 \%$ & 122 & $42.7 \%$ \\
\hline DA & 71.56 & $0.1 \%$ & 73.5 & $4.9 \%$ \\
\hline
\end{tabular}

In the second scenario, the proposed and previous methods, the PID control mode is tuned in PID at both primary and secondary loop. The result comparisons of proposed and previous methods are tabulated in the Table 4.

TABLE IV. PARAMETER PID USING PID-PID

\begin{tabular}{|c|c|c|c|c|c|c|}
\hline Methods & Kp & Ki & Kd & Kps & Kis & Kds \\
\hline Lee et al. & 5.83 & 0.055 & 27.984 & 3.444 & 0.166 & 2.221 \\
\hline $\begin{array}{c}\text { Luus \& } \\
\text { Jaloka }\end{array}$ & 2.907 & 0.032 & 11.738 & 1.880 & 0.313 & 1.367 \\
\hline GA & 5.972 & 0.058 & 9.274 & 3.908 & 0.784 & 0.140 \\
\hline DA & 4.892 & 0.049 & 1.674 & 2.339 & 0.919 & 3.795 \\
\hline $\begin{array}{c}\text { Zieger } \\
\text { Nichols }\end{array}$ & 5.37 & 0.1568 & 10 & 3.45 & 0.4 & 2.56 \\
\hline
\end{tabular}

By utilizing the tuning parameters in Table 4, the simulation result of cascade control in secondary and primary control can be shown in Fig. 4 and Fig. 5, respectively.

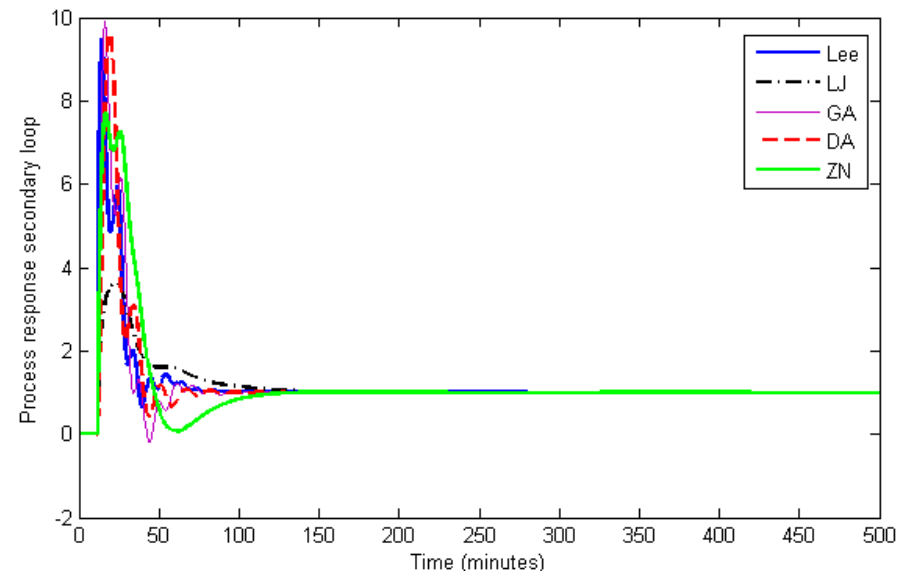

Fig. 4. Closed loop responses for secondary loop using PID-PID

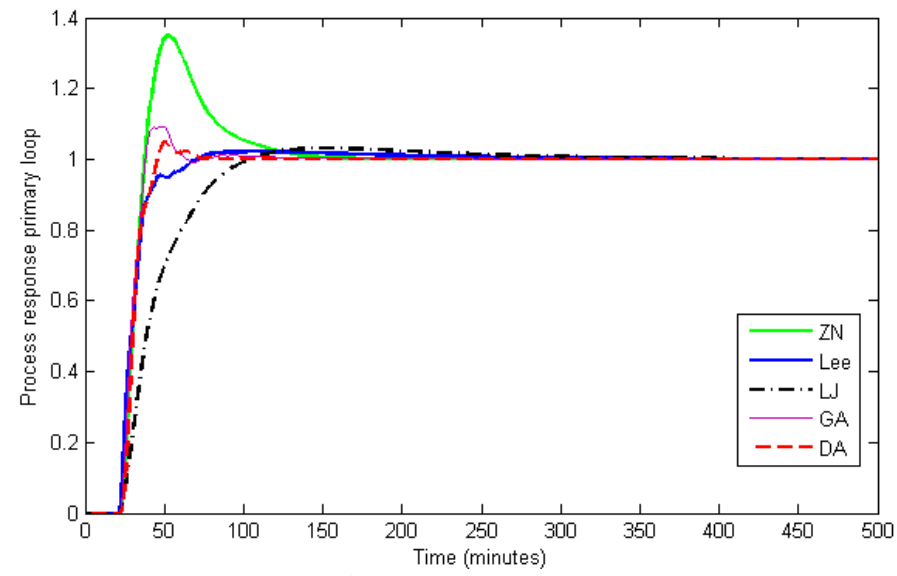

Fig. 5. Closed loop responses for primary loop using PID-PID

From the Fig. 5, it is obtained the quantitative performance (IAE) and qualitative performance of the system, and it is tabulated on the Table 5. The result of simulation, tuning PIDDA surpass all previous method and it is shown in the smallest IAE and fastest settling time. Moreover, the method proposed by Lee and Luus \& Jaluka obtain sluggish response and it is shown by smaller overshoot.

TABLE V. PERFORMANCE SYSTEM MODE PID-PID

\begin{tabular}{|c|c|c|c|c|}
\hline Metode & IAE & $\begin{array}{c}\text { Error } \\
\text { steady state }\end{array}$ & $\begin{array}{c}\text { Settling } \\
\text { Time }\end{array}$ & $\begin{array}{c}\% \\
\text { Overshoot }\end{array}$ \\
\hline Lee et al. & 67.50 & $0.1 \%$ & 131.5 & $2.2 \%$ \\
\hline $\begin{array}{c}\text { Luus \& } \\
\text { Jaloka }\end{array}$ & 95.42 & $0.1 \%$ & 216.5 & $3.1 \%$ \\
\hline GA & 63.77 & $0.0 \%$ & 74 & $9.4 \%$ \\
\hline DA & 62.88 & $0.0 \%$ & 66.5 & $4.8 \%$ \\
\hline $\begin{array}{c}\text { Zieger } \\
\text { Nichols }\end{array}$ & 89.36 & $0.0 \%$ & 107.5 & $39.7 \%$ \\
\hline
\end{tabular}




\section{CONCLUTION}

Duelist Algorithm is used to optimize the tuning parameters of PID controller on secondary and primary loops. IAE is utilized as objective function of the optimization. The results of the proposed method are compared with the previous method i.e. Zieger Nichols, proposed method by Lee et al., proposed method by Luus \& Jaloka, and GA. The simulation results show, the PID-DA auto tuning provides the best performance. It can be concluded that the PID-DA auto tuning method provide more robust performance of the control system.

\section{ACKNOWLEDGMENT}

The author gratefully thanks to PT. PJB UP Paiton and Institut Teknologi Sepuluh Nopember (ITS) Surabaya for providing the facilities for conducting this research. Also thanks to PT PETROKIMIA GRESIK for supporting on this research.

\section{REFERENCES}

[1] A. Bagis, "Determination of the PID Controller Parameters by Modified Genetic Algorithm for Improved Performance," Inf. Sci. Eng., vol. 1480, pp. 1469-1480, 2007.

[2] M. Luyben and W. L. Luyben, No Title. Singapore: McGraw-Hill, 1997.

[3] D. E. Seaborg, T. F. Edgar, and D. A. Mellichamp, Process Dynamics and Control. United States of America: Jhon Wiley \& Sons, Inc, 2004.

[4] I. Kaya, "Improving performance using cascade control and a Smith predictor," ISA Trans., vol. 40, pp. 223-234, 2001.

[5] T. Liu, D. Gu, and W. Zhang, "Decoupling two-degree-of-freedom control strategy for cascade control systems," J. Process Conrol, vol. 15, pp. 159-167, 2005.

[6] I. Kaya, N. Tan, and D. P. Atherton, "Improved cascade control structure for enhanced performance _," Process Control, vol. 17, pp. 3-16, 2007.

[7] S. Song, W. Cai, and Y. Wang, "Auto-tuning of cascade control systems," ISA Trans., vol. 42, pp. 63-72, 2003.

[8] K. K. Tan, T. H. Lee, and R. Ferdous, "Simultaneous online automatic tuning of cascade control for open loop stable processes," ISA Trans., vol. 39, pp. 233-242, 2000.

[9] J. Jeng and M. Lee, "Simultaneous automatic tuning of cascade control systems from closed-loop step response data," J. Process Control, vol. 22, no. 6, pp. 1020-1033, 2012.

[10] M. Veronesi and A. Visioli, "Simultaneous closed-loop automatic tuning method for cascade controllers," in IET Control Theory and Applications, 2011, no. June 2010, pp. 263-270.

[11] J. Jeng, "Simultaneous closed-loop tuning of cascade controllers based directly on set-point step-response data," J. Process Control, vol. 24 , no. 5 , pp. $652-662,2014$.

[12] M. V. Sadasivarao and M. Chidambaram, "PID Controller tuning of cascade control systems using genetic algorithm," pp. 343-354, 2006.

[13] T. R. Biyanto, H. Y. Fibrianto, E. Listijorini, and T. Budiati, "Duelist Algorithm: An Algorithm Inspired by How Duelist Improve Their Capabilities in a Duel," in The Seventh International Conference on Swarm Intelegence, 2015. 